\title{
Multidimensional structure of chiral crystals in quark matter
}

\section{Tong-Gyu Lee*}

Department of Physics, Kyoto University, Kyoto 606-8502, Japan

Department of Natural Science, Kochi University, Kochi 780-8520, Japan

E-mail: tonggyu.lee@yukawa.kyoto-u.ac.jp

\section{Nobutoshi Yasutake}

Department of Physics, Chiba Institute of Technology, Chiba 275-0023, Japan

E-mail: nobutoshi.yasutake@it-chiba.ac.jp

\section{Toshiki Maruyama}

Advanced Science Research Center, Japan Atomic Energy Agency, Ibaraki 319-1195, Japan

E-mail: maruyama.toshiki@jaea.go.jp

\section{Toshitaka Tatsumi}

Department of Physics, Kyoto University, Kyoto 606-8502, Japan

E-mail: tatsumieruby.scphys.kyoto-u.ac.jp

We discuss the possibility of a multidimensional structure of inhomogeneous chiral condensates, which might be realized in cold and dense quark matter or the core of compact stars. For onedimensional structures, the system becomes unstable at finite temperature due to the LandauPeierls instability attributed to the excitation of the Nambu-Goldstone modes, with the result that there is no long-range order, while quasi-long-range order is realized instead. On the other hand, chirally inhomogeneous phases with two- or three-dimensional modulations may be realized as a true long-range ordered phase at any temperature, as inferred from the Landau-Peierls theorem. We here consider possible strategies to explore a multidimensional structure of chiral crystals.

The 26th International Nuclear Physics Conference

11-16 September, 2016

Adelaide, Australia

\footnotetext{
* Speaker.
} 


\section{Introduction}

The determination of a phase diagram for quantum chromodynamics (QCD) in the plane of temperature and baryon chemical potential is among the ultimate goals in nuclear physics, both experimentally and theoretically (see, e.g., [1] for a recent review). In contrast to, however, a growing understanding of the high temperature regime via ultrarelativistic heavy-ion collisions together with ab initio lattice QCD simulations, the finite density regime is still less well understood due in part to the so-called sign problem. For the experimental exploration of this regime, the socalled beam energy scans at RHIC or future experiments prepared at FAIR, NICA, and J-PARC have attracted attention, and at the same time the challenge of extracting the properties of dense QCD matter from the data obtained in such experiments has stared. On the theoretical side, the corresponding regime is expected to be very rich. In particular, in recent studies of the QCD phase diagram at nonvanishing baryon density, various inhomogeneous phases have been discussed in the context of chiral symmetry (for a comprehensive review see [2] and references therein), and such phases have been suggested to appear in the cold and dense regime of the QCD phase diagram within some effective models [3, 4, 5] or Dyson-Schwinger equation approach [6] (see, e.g., Fig. 11. Such a regime may be relevant for compact stars, so that inhomogeneous chiral phases may possibly exist in the core of such stars and thus might have astrophysical implications 17,8 , 9. In addition, since the presence of inhomogeneous phases can give rise to a modification of the conventional QCD phase diagram, where inhomogeneous chiral phases emerge instead of the conventional first-order chiral transition line and therefore the chiral transition region is extended, it may have phenomenological implications for heavy-ion collisions as well.

The inhomogeneous chiral phase is characterized by a space-dependent but time-independent order parameter $\phi(\vec{x})$, whose general form consists of the scalar and pseudoscalar condensates: $\phi(\vec{x}) \equiv\langle\bar{\psi} \psi\rangle(\vec{x})+i\left\langle\bar{\psi} i \gamma_{5} \tau_{a} \psi\right\rangle(\vec{x}) \delta_{a 3}=\Delta(\vec{x}) e^{i \theta(\vec{x})}$, where $\psi$ is a light quark field, $\tau_{a}$ are the three Pauli matrices in flavor space, $\Delta(\vec{x})$ is a space-dependent amplitude, and $\theta(\vec{x})(=\vec{q} \cdot \vec{x})$ represents a

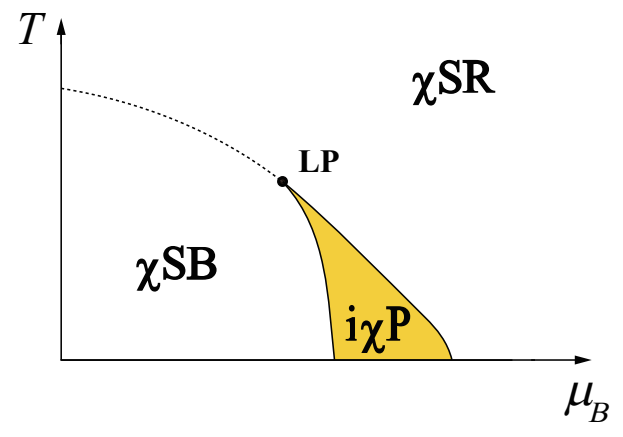

Figure 1: Schematic phase diagram for chiral symmetry at finite temperature $T$ and baryon chemical potential $\mu_{B}$. The yellow region enclosed by two solid lines (phase boundaries), starting from the Lifshit point (LP), shows inhomogeneous chiral phases (i $\chi \mathrm{P})$, where chiral symmetry breaking is spatially inhomogeneous. The left boundary is of first or second order, depending on the type of $\mathrm{i} \chi \mathrm{P}$ [3] 5 , while the right one is universally of second order. The dotted line represents the boundary between the spatially homogeneous chiral symmetry breaking $(\chi \mathrm{SB})$ and the chiral symmetry restored $(\chi \mathrm{SR})$ phase, which is of second order in the chiral limit. 
spatially modulated phase with finite wavevector $\vec{q}$. For one-dimensional (1D) modulations (e.g., let $z$ be the modulation direction, that is, $\vec{q} \cdot \vec{x}=(0,0, q z))$, there are two well-known ground states of inhomogeneous phases in the context of condensed matter physics: one is a Fulde-Ferrell (FF) type condensate characterized by a modulating phase with constant amplitude, $\phi(z)=\Delta e^{i \theta(z)}$, and the other is the Larkin-Ovchinnikov (LO) type condensate characterized by a modulating amplitude, $\phi(z)=\Delta(z)$. In the context of quark matter, the former corresponds to the dual chiral density wave (DCDW) [3], while the latter the real kink crystal (RKC) [4, 5]. Quarkyonic chiral spirals also are of the former type in the context of quarkyonic matter [10]. These chiral modulations are one dimensional in space and based on analytically known solutions for 1+1D systems [11], which are embedded in three spatial dimensions. Within the mean-filed approximation, the RKC is more favorable in energy than the DCDW. This situation, however, is reversed in a background magnetic field (see, e.g., [2]).

While so far most studies of inhomogeneous chiral condensates have been devoted to 1D modulations, a multidimensional modulation may also be realized. In the following, we will discuss the properties of inhomogeneous chiral phases with 1D modulations beyond the mean-field level, and then consider the possibility of a multidimensional structure of chiral crystals.

\section{One-dimensional structure of chiral crystals}

We consider a phase with a 1D modulation of the chiral condensate and discuss its stability against low energy fluctuations about the order parameter, such as the Nambu-Goldstone (NG) modes associated with spontaneous symmetry breaking. In the inhomogeneous chiral phase, in general, not only the $S U(2)_{L} \times S U(2)_{R}$ chiral symmetry but also the translational and rotational symmetries are spontaneously broken, but only three [12] or four [13] independent NG modes appear due to redundancies of broken global spacetime symmetries in addition to a locking of flavor and translational symmetries.

For definiteness, let us consider here the inhomogeneous chiral condensate of the DCDW type [3 12]. Assuming that $\phi(z)$ is a real four-component field, we can define the DCDW ground state as $\phi_{0}(z)=\Delta(\cos \theta(z), 0,0, \sin \theta(z))^{T} \equiv\left(\sigma(z), 0,0, \pi_{3}(z)\right)^{T}$ with $\sigma(z) \equiv\langle\bar{\psi} \psi\rangle(z)$ and $\pi_{3}(z) \equiv$ $\left\langle\bar{\psi} i \gamma_{5} \tau_{3} \psi\right\rangle(z)$, where $\theta(z)=q z$ for the 1D modulation (see below, Fig. 2). We then introduce general fluctuations around $\phi_{0}(z)$ using the $S^{3}$ parametrization being $\phi=(\Delta+\delta) U\left(\beta_{i}\right) \phi_{0}$ with $U\left(\beta_{i}\right) \equiv$

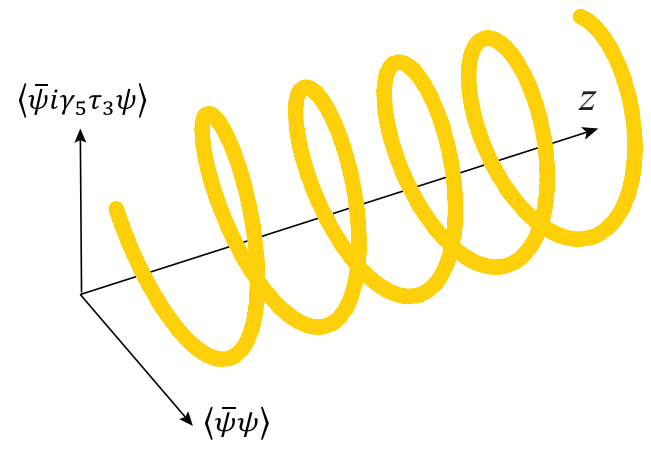

Figure 2: Schematic illustration of the configuration of the DCDW condensate [3, 12]. 
$\Pi_{i=1}^{3} e^{i \beta_{i} L_{i}}$ [12], where the parameters $\delta, \beta_{i}$, and $L_{i}$ correspond to an amplitude fluctuation, a rotation in the 4D sphere of the chiral circle, and the $O(4)$ generators, respectively. This, to leading order in fluctuations, yields $\phi(z)=(1+\delta) \phi_{0}(z)+\left(-\Delta \beta_{3} \sin q z, \Delta \beta_{1} \cos q z, \Delta \beta_{2} \cos q z, \Delta \beta_{3} \cos q z\right)^{T}+$ $O\left(\beta_{i}^{2}, \delta^{2}, \delta \beta_{i}\right)$, which illustrates the fluctuations corresponding to both the NG and Higgs modes. Promoting these parameters to fields, i.e., $\delta \rightarrow \delta(t, \vec{x})$ and $\beta_{i} \rightarrow \beta_{i}(t, \vec{x})$, we can take into account the local fluctuations, so that a low energy effective theory for such fluctuations can be derived in a systematic manner within the Gaussian approximation. From this, we can obtain the dispersion relation of the massless modes $\omega_{03}$ involving $\delta$ and $\beta_{3}$, together with that of the modes $\omega_{12}$ involving $\beta_{1}$ and $\beta_{2}$. The former takes the form $\omega_{03}^{2}=A k_{z}^{2}+B\left(\vec{k}^{2}\right)^{2}+C \vec{k}^{2} k_{z}^{2}+D k_{z}^{4}$, while the latter $\omega_{12}^{2}=a k_{z}^{2}+b\left(\vec{k}^{2}\right)^{2}$, which are spatially anisotropic and particularly soft in directions normal to the modulation (i.e., $x$ - $y$ directions) due to the absence of quadratic terms in the transverse momentum (i.e., terms with $k_{x}^{2}$ and $k_{y}^{2}$ ). The coefficients $A, B, C, D, a$, and $b$ depend on the Ginzburg-Landau coefficients in addition to the variational parameters, $\Delta$ and $q$, (see [12] for details of the coefficients), which can be evaluated within chiral effective models.

Such low energy modes with the dispersions mentioned above impact on the condensed phase of the DCDW type and consequently wash out the order parameter, i.e., $\langle\phi(z)\rangle=\left\langle U\left(\beta_{i}\right) \phi_{0}(z)\right\rangle=$ 0 , where $\langle\cdots\rangle$ means the thermal average, owing to the softening of the fluctuations in the $x-y$ directions (strictly speaking, the logarithmic divergence of Gaussian fluctuations in the infrared region). The same applies to the case of the RKC condensate [13], whose ground state takes the form $\phi_{0}(z)=(c \sqrt{v} \operatorname{sn}(c z \mid v), 0,0,0) \equiv(\sigma(z), 0,0,0)$, where sn is a Jacobian elliptic function with the modulus $v \in[0,1]$, and $c$ is a scale related to the maximum mean-field value of $\phi_{0}(z)$ and also is a function of $v$. Therefore, the 1D structure of such inhomogeneous phases is unstable and eventually disappears at finite temperature, which is termed the Landau-Peierls instability [14].

However, although a disordered phase is usually characterized by exponential decays of the order parameter correlation function at large distances, the present system exhibits a power-law decay of the spatial correlations [12]: $\left\langle\phi\left(z \vec{e}_{z}\right) \phi(0)\right\rangle \propto \cos q z\left(z / z_{0}\right)^{-\eta(T)}$ for the $z$ direction parallel to the modulation, while $\left\langle\phi\left(x_{t} \vec{e}_{t}\right) \phi(0)\right\rangle \propto\left(x_{t} / x_{0}\right)^{-2 \eta(T)}$ for the $x-y$ directions transverse to the modulation, where $x_{t}$ is a transverse distance, $\vec{e}$ is an unit vector in the spatial direction, the exponent depending temperature, $\eta(T)\left(=T / T_{0}\right)$, corresponds to the so-called Caillé exponent, and all $z_{0}$, $x_{0}$, and $T_{0}$ can be determined within the effective model. This algebraic behavior of the correlation function is known as quasi-long-range order. Thus, the inhomogeneous chiral phase with a 1D periodic structure may survive in a quasi-1D ordered form [12]13] even at finite temperature, as in the smectic-A phases of liquid crystals (see, e.g., [15] for a review of smectic states). Therefore, such a phase should be distinguished from a disordered phase, albeit not true long-range order.

On the other hand, there are some possibilities to suppress the Landau-Peierls instability, which may lead to a long-range ordered phase. Firstly, in the case of the $T=0$ limit, quantum fluctuations are not so strong as to wash out the order parameter, that is, the system is relatively stable, so that the DCDW phase at vanishing temperature is expected to truly exist as a long-range ordered phase. Also, while this phase is critical at $T>0$ due to quasi-long-range order, it may be practically realized also in the very low-temperature region of $T<T_{0}$. Secondly, unlike the infinite system, the finite system, where the range of the order-parameter correlations exceeds the system size, can permit a 1D structure of the inhomogeneous condensate. The reason why the inhomogeneous phase with 1D modulation is unstable at nonzero temperatures is that Gaussian fluctuations 
are logarithmically divergent in the long-wavelength limit owing to the soft modes in the transverse direction (i.e., transverse momentum integral diverges in the infrared region), so that one can introduce an infrared cutoff for the regularization and regard it as a transverse scale of the system. If the length scale of the fluctuations is much larger than the system size, the 1D structure of the condensate can remain finite (i.e., finite-size effects) [13, 16]. Similarly, the algebraic (quasi-long-range) correlations can effectively emulate true long-range order, depending on the experimental resolution [16 15], where one can distinguish the Bragg peaks (long-range order) from quasi-Bragg peaks (algebraic order) via very high-resolution measurements. Thirdly, since in general external magnetic fields $(B)$ explicitly break the rotational symmetry of the system, the dispersion relation of the NG modes associated with spontaneously broken symmetries can be changed in the inhomogeneous chiral phase under the situation of $B \neq 0$. As already mentioned above, there exists a spatial anisotropy of the dispersion relation in the DCDW phase due to the global rotational symmetry of the system, and this stems from the lack of the terms with $k_{x}^{2}$ and $k_{y}^{2}$. Thus, in an external magnetic field, such terms would make a comeback depending on $B$, which gives rise to an improvement for the softening of the transverse fluctuations and hence leads to a stable inhomogeneous phase with a 1D modulation. Finally, if the condensate modulation is two- or three-dimensional in space, since the soft directions of the condensate decrease compared to those of the 1D condensate, the suppression of the infrared singularity of Gaussian fluctuations can be expected [14]. Therefore, the stabilization occurs and true long-range order can be realized. Here we focus on this last topic.

\section{Multidimensional structure of chiral crystals}

We now consider the possibility of a multidimensional chiral crystal. Although it would be interesting to see whether a phase with true long-range order in 2D or 3D modulations is energetically favored over one with quasi-long-range order in 1D modulations, we restrict ourselves only to true long-range ordered phases here. To determine whether a multidimensional modulation is thermodynamically favored, one must compare its free energy with that of 1D modulation. However, in contrast to purely 1+1D systems where analytic solutions are known [11], analytic solutions for $2+1 \mathrm{D}$ or $3+1 \mathrm{D}$ systems have yet to be discovered. Thus, one often assumes possible ansätze for $2 \mathrm{D}$ or $3 \mathrm{D}$ modulations.

In the vicinity of the Lifshitz point (LP), a Ginzburg-Landau analysis using some ansätze for multidimensional LO-type real condensates [17], together with the 1D solitonic-type (RKC) and 1D FF-type (DCDW) condensates, concludes that the RKC condensate is the most favored, followed in order by the 1D LO-type, 2D LO-type, 3D LO-type, and DCDW-type condensates. It is interesting to note here that within multidimensional FF-type complex condensates the most favored condensate is the 2D condensate, but not the $1 \mathrm{D}$ one (i.e., DCDW). A similar result holds for the case of vanishing temperature; a Nambu-Jona-Lasinio (NJL) model calculation at $T=0$ using two 2D LO-type ansätze (square and hexagonal lattices) [18] shows that the 2D modulations are disfavored against the $1 \mathrm{D}$ ones (i.e., RKC). Thus, at least around the LP or at zero temperature, 1D modulations are found to be favored compared with 2D or 3D ones. However, a phase with multidimensional modulation may be realized in different areas of inhomogeneous chiral phases. Actually, remarkable studies are reported in a variety of systems [19, 20, 21, 22, 23], where the formation of a multidimensional crystalline structure is predicted. On the other hand, interestingly, an 
NJL-model analysis at high density region shows that 2D modulations tend to be favored against 1D ones [18]. In such a region, it has been suggested that the so-called inhomogeneous continent may appear [24, 25, 26], which means that 2D structures could be formed at high densities. However, at present, a definite understanding of this region have yet to be obtained.

Another possible approach to find a multidimensional chiral crystal, in particular outside the vicinity of the LP and the region at $T=0$, is to employ the Thomas-Fermi approximation (TFA) [19]. Using the formula for single-particle energy levels $\left(E_{T F}\right)$ obtained in the TFA, we can numerically investigate whether the free energy for a given ansätz of the multidimensional modulation is energetically favored, as with the case of the GL analysis [17]. Here, the expression of $E_{T F}$ should include the gradient term of the condensate $M(\vec{x})\left(=\Delta(\vec{x}) e^{i \theta(\vec{x})}\right)$, but there is no such term in the conventional NJL model. Thus, we need to effectively extract the derivative coupling term (e.g., a term with $\nabla M(\vec{x})$, in particular $\nabla \Delta(\vec{x}))$ in $E_{T F}$ by a good scale transformation, as in a local chiral transformation $\left(\psi \rightarrow e^{i \gamma_{5} \theta(\vec{x}) / 2} \psi\right)$ which leads to a momentum-dependent interaction term (e.g., a term with $\nabla \theta(\vec{x}))$ in the formula for the energy spectrum (see [3] for the DCDW with $\nabla \theta(z)=q)$. In this study, we have assumed the shape of the condensate function $M(\vec{x})$ as a specific ansätz, so that there is no self-consistency between $E_{T F}$ and $M(\vec{x})$. This goes for the GL and NJL-model analyses. Therefore, in general, we should explore the structure of $M(\vec{x})$ without any assumption.

We thus consider here a self-consistent way to numerically explore a multidimensional structure of chiral crystals without assuming an ansätz for the condensate function. Within the NJL model, one can obtain the self-consistency condition on $M(\vec{x}): M(\vec{x}) \propto \sum_{E_{n}} \tanh \left(\frac{E_{n}-\mu}{2 T}\right) \bar{\psi}(\vec{x})(1-$ $\left.\gamma^{5}\right) \psi(\vec{x})$ [5]. Here, by discretizing the effective Dirac equation $\left(H_{D} \psi_{i}=E_{i} \psi_{i}\right)$ with the Hamiltonian $H_{D}=-i \gamma^{0} \gamma^{i} \partial_{i}+\gamma^{0} M(\vec{x})$, the eigenvalues $\left\{E_{i}\right\}$ and the corresponding eigenvectors (wave functions) $\left\{\psi_{i}\right\}$ for eigenstates $\{i\}$ can be determined simultaneously and numerically. Using these obtained values, the possible phase diagram can be derived from the estimation of the thermodynamic potential via the energetically favored $M(\vec{x})$. A similar approach without any specific ansätz has been recently presented employing the finite-mode approach [26], where the inhomogeneous condensate is written as a superposition of a finite number of plane waves. Before investigating an arbitrary $M(\vec{x})$ with 2D or 3D spatial modulations, we first need to correctly reproduce the wellknown results in 1+1D systems [11] to check the validity of our method. Thus, taking into account the 1D condensate (RKC or chiral spiral), we demonstrate whether the shape of the numerically obtained $M(x)$ becomes close to analytic solutions, such as the Jacobi sn elliptic function or plane wave solution. By discretizing the 1+1D Dirac equation using the finite-difference method (e.g., $\left.\partial_{x} f(x) \equiv \Delta f=\left(f_{i+1}-f_{i-1}\right) / 2 \Delta x\right)$ and then solving the corresponding nonlinear complex eigenvalue problem satisfying the shape of energy spectrum in purely $1+1$ dimensions (symmetric for the RKC, while asymmetry for the chiral spiral), we can obtain the respective $E_{i}$ and $\psi_{i}=\left(f_{i}, g_{i}\right)$. Eventually, the Gross-Neveu type phase diagram [11] can be obtained, and then the investigation toward 2D chiral crystals is permitted.

\section{Summary and outlook}

We discussed the properties of inhomogeneous chiral phases with 1D spatial modulations. In particular, we focused on the stability of 1D modulations against fluctuations. Consequently, the 1D 
structure is unstable at finite temperature due to the NG mode excitations, and therefore there is no true long-range order, albeit being quasi-long-range order. One of factors to evacuate this instability is two- or three-dimensional modulations, as inferred from the Landau-Peierls theorem. We then argued the possibility of a multidimensional chiral crystal. While some studies have been showed that $1 \mathrm{D}$ modulations are the most favored, a multidimensional modulation in a region other than that around LP and of $T=0$ may become energetically preferred over 1D modulations. Of cause, whether higher-dimensional modulations are favored over quasi-long-range order is nontrivial, so that it would also be worthwhile to compare their free energies. This is still an open question. On the other hand, for the search for a multidimensional structure of chiral crystals, we presented some possible strategies: one is a numerical approach using the TFA, and the other is a self-consistent way without any assumption on the shape of the condensate functions. The latter, in particular, may further need to a reduction of computational costs or a major breakthrough in solving a nonlinear eigenvalue problem with a complicated matrix in an effort to study a multidimensional structure.

It would be interesting to consider Coulomb interactions in the system, which may lead to pasta structures (chiral pastas). For phenomenological implications, it may be also worthwhile to investigate whether or not there appear pasta structures (as in hadron-quark mixed phases [27) at zero temperature in general and gyroid structures [28] at finite temperature in particular. Detailed discussion on this direction remains as a future problem.

\section{Acknowledgments}

This work is partially supported by Grant-in-Aid for Scientific Research on Innovative Areas through No. 24105008 provided by MEXT.

\section{References}

[1] K. Fukushima and C. Sasaki, The phase diagram of nuclear and quark matter at high baryon density, Prog. Part. Nucl. Phys. 72(2013) 99-154, 1301.6377.

[2] M. Buballa and S. Carignano, Inhomogeneous chiral condensates, Prog. Part. Nucl. Phys. 81 (2015) 39-96 [1406.1367].

[3] E. Nakano and T. Tatsumi, Chiral symmetry and density wave in quark matter, Phys. Rev. D71 (2005) 114006 hep-ph/0411350.

[4] D. Nickel, How many phases meet at the chiral critical point?,Phys. Rev. Lett. 103 (2009) 072301 0902.1778 .

[5] D. Nickel, Inhomogeneous phases in the Nambu-Jona-Lasino and quark-meson model, Phys. Rev. D80 (2009) 074025 0906.5295.

[6] D. Muller, M. Buballa and J. Wambach, Dyson-Schwinger study of chiral density waves in QCD, Phys. Lett. B727 (2013) 240-243, [1308.4303.

[7] T. Tatsumi and T. Muto, Quark beta decay in the inhomogeneous chiral phase and cooling of compact stars, Phys. Rev. D89(2014) 103005 [1403.1927].

[8] S. Carignano, E. J. Ferrer, V. de la Incera and L. Paulucci, Crystalline chiral condensates as a component of compact stars, Phys. Rev. D92 (2015) 105018. 1505.05094]. 
[9] M. Buballa and S. Carignano, Inhomogeneous chiral symmetry breaking in dense neutron-star matter, Eur. Phys. J. A52 (2016) 57, [1508.04361].

[10] T. Kojo, Y. Hidaka, L. McLerran and R. D. Pisarski, Quarkyonic chiral spirals, Nucl. Phys. A843 (2010) 37-58, 0912.3800.

[11] G. Basar, G. V. Dunne and M. Thies, Inhomogeneous condensates in the thermodynamics of the chiral NJL(2) model, Phys. Rev. D79 (2009) 105012, 0903.1868.

[12] T.-G. Lee, E. Nakano, Y. Tsue, T. Tatsumi and B. Friman, Landau-Peierls instability in a Fulde-Ferrell type inhomogeneous chiral condensed phase, Phys. Rev. D92 (2015) 034024 [1504.03185.

[13] Y. Hidaka, K. Kamikado, T. Kanazawa and T. Noumi, Phonons, pions and quasi-long-range order in spatially modulated chiral condensates, Phys. Rev. D92 (2015) 034003 [1505.00848].

[14] L. D. Landau and E. M. Lifshitz, Statistical Physics. Pergamon Press, Oxford 1969.

[15] W. H. de Jeu, B. I. Ostrovskii and A. N. Shalaginov, Structure and fluctuations of smectic membranes, Rev. Mod. Phys. 75 (2003) 181-235

[16] G. Baym, B. L. Friman and G. Grinstein, Fluctuations and long-range order in finite-temperature pion condensates, Nucl. Phys. B210 (1982) 193-209.

[17] H. Abuki, D. Ishibashi and K. Suzuki, Crystalline chiral condensates off the tricritical point in a generalized Ginzburg-Landau approach, Phys. Rev. D85 (2012) 074002, [1109.1615.

[18] S. Carignano and M. Buballa, Two-dimensional chiral crystals in the NJL model, Phys. Rev. D86 (2012) 074018 [1203.5343].

[19] A. B. Migdal, O. A. Markin and I. N. Mishustin, Pion interaction in nuclear matter and $\pi$ condensation, JETP 43 (1976) 830.

[20] H. Shimahara, Structure of the fulde-ferrell-larkin-ovchinnikov state in two-dimensional superconductors, Journal of the Physical Society of Japan 67 (1998) 736-739. arXiv:cond-mat/9711017.

[21] J. A. Bowers and K. Rajagopal, The crystallography of color superconductivity, Phys. Rev. D66 (2002) 065002 "hep-ph/0204079.

[22] T. Kojo, Y. Hidaka, K. Fukushima, L. D. McLerran and R. D. Pisarski, Interweaving chiral spirals, Nucl. Phys. A875 (2012) 94-138, [1107.2124].

[23] G. Cao and A. Huang, Solitonic modulation and Lifshitz point in an external magnetic field within Nambu-Jona-Lasinio model, Phys. Rev. D93 (2016) 076007, 1601.03493.

[24] S. Carignano and M. Buballa, Inhomogeneous islands and continents in the Nambu-Jona-Lasinio model, Acta Phys. Polon. Supp. 5 (2012) 641-658, [111.4400.

[25] S. Carignano, M. Buballa and B.-J. Schaefer, Inhomogeneous phases in the quark-meson model with vacuum fluctuations, Phys. Rev. D90 (2014) 014033, 1404.0057.

[26] A. Heinz, F. Giacosa, M. Wagner and D. H. Rischke, Inhomogeneous condensation in effective models for QCD using the finite-mode approach, Phys. Rev. D93 (2016) 014007, [1508.06057.

[27] N. Yasutake, T. Maruyama and T. Tatsumi, Hot hadron-quark mixed phase including hyperons, Phys. Rev. D80 (2009) 123009, 0910.1144.

[28] K. Nakazato, K. Oyamatsu and S. Yamada, Gyroid Phase in Nuclear Pasta, Phys. Rev. Lett. 103 (2009) 132501 0905.2016. 